\title{
Avaliação Neurológica Realizada por Enfermeiros em Vítimas de Traumatismo Cranioencefálico
}

\author{
Neurological Assessment for Nurses In Victims of Traumatic Brain Injury \\ Camila Fernandes de Amorim ${ }^{1}$, João Evangelista Menezes Júnior ${ }^{2}$, Thiago \\ Enggle de Araújo Alves', Dayane Pessoa de Araújó, Fausto Pierdoná \\ Gúzen ${ }^{5}$, José Rodolfo Lopes de Paiva Cavalcanti ${ }^{6}$
}

\begin{abstract}
RESUMO
Objetivo. analisar a compreensão dos enfermeiros que atuam em Unidade de Terapia Intensiva (UTI) acerca da avaliação neurológica em vítimas de Traumatismo Cranioencefálico (TCE). Método. trata-se de uma pesquisa descritiva e de abordagem qualitativa, realizada com sete enfermeiros que atuam na UTI de um hospital público de Mossoró-RN. Os dados foram coletados a partir de entrevistas semiestruturadas que foram discutidas à luz da análise de conteúdo. A pesquisa só teve início após aprovação pelo comitê de ética institucional mediante protocolo n. 107/10 e CAAE n. 2973.0.000.351-10. Resultados. percebeu-se que a maioria dos Enfermeiros considerava a avaliação neurológica indispensável no cuidado do paciente com TCE. Porém, se limitavam à aplicaçáo da Escala de Coma de Glasgow, assim como havia a falta de padronização nessa assistência, transformando-a em procedimentos tipicamente individuais. Conclusáo. os resultados apontados no estudo sugerem a limitaçáo do conhecimento acerca da avaliação neurológica aplicada às vítimas de TCE's, por parte dos enfermeiros do setor estudado.
\end{abstract}

Unitermos. Neurologia, Traumatismos Encefálicos, Avaliação em Enfermagem.

Citaçáo. Amorim CF, Menezes Júnior JE, Alves TEA, Araújo DP, Gúzen FP, Cavalcanti JRLP. Avaliação Neurológica Realizada por Enfermeiros em Vítimas de Traumatismo Cranioencefálico.

\begin{abstract}
Objective. to analyze the understanding of nurses working in intensive care unit (ICU) on the neurological evaluation in traumatic brain injury (TBI). Method. it is a descriptive and qualitative approach, held in a public hospital in Mossoro, Rio Grande do Norte, Brazil. The sample consisted of seven nurses working in ICU. Data were collected from semi-structured interviews that were discussed in basis of the content analysis. The research began only after approval by the institutional Ethics Committee under the Protocol number 107/10 and CAAE n. 2973.0.000.351-10. Results. the majority of nurses considered essential in evaluating neurological care for patients with TBI. But they were limited to applying the Glasgow Coma Scale, as was the lack of standardization in this service, making in a typical individual procedures. Conclusion. The results in this study suggest the limitation of knowledge about the neurological assessment applied to TBI's, by the nurses studied in this health unit.
\end{abstract}

Keywords. Neurology, Brain Injuries, Nursing Assessment.

Citation. Amorim CF, Menezes Júnior JE, Alves TEA, Araújo DP, Gúzen FP, Cavalcanti JRLP. Neurological Assessment for Nurses In Victims of Traumatic Brain Injury.
Estudo realizado no Hospital Regional Tarcísio Maia (HRTM), Mossoró-RN, pelo Laboratório de Neurologia Experimental - Departamento de Ciências Biomédicas - Universidade do Estado do Rio Grande do Norte (UERN), Mossoró-RN, Brasil.

1.Enfermeira, especialista em Urgência e Emergência, Mossoró-RN, Brasil. 2.Enfermeiro, especialista em Enfermagem do Trabalho, Mossoró-RN, Brasil. 3.Enfermeiro, mestre em Enfermagem (UFRN), docente da Faculdade de Enfermagem Nova Esperança de Mossoró (FACENE/RN), Mossoró-RN, Brasil. 4.Enfermeira, mestre em farmacologia (UFC), docente da Universidade do Estado do Rio Grande do Norte, Mossoró-RN, Brasil.

5.Farmacêutico, doutor em Psicobiologia (UFRN), docente da Universidade do Estado do Rio Grande do Norte, Mossoró-RN, Brasil.

6.Enfermeiro, mestre em Psicobiologia (UFRN), docente da Universidade do Estado do Rio Grande do Norte, Mossoró-RN, Brasil.
Endereço para correspondência: José Rodolfo Lopes de Paiva Cavalcanti Rua Atirador Miguel Antônio da Silva Neto, S/N, Bairro Aeroporto. CEP 59607-360, Mossoró-RN, Brasil.

Telefone: (84) 8818-7226

E-mail: joserodolfolopes@uol.com.br 


\section{INTRODUÇÃO}

O traumatismo tem despontado entre as três principais causas de morte na população em geral, perdendo apenas para as doenças cardiovasculares e as neoplásicas. Nos adultos jovens, isto é, nos sujeitos abaixo dos quarenta anos de idade, ocupa a primeira colocação entre as taxas de mortalidade, sendo o TCE o seu maior representante $e^{1-3}$.

De uma maneira geral, o TCE é definido como toda agressão que ocasiona dano anatômico ou comprometimento funcional do couro cabeludo, ossos do crânio, meninges ou encéfalo. Sua etiologia está relacionada à lesão traumática provocada por acidentes de trânsito, homicídios, suicídios, agressóes interpessoais e demais tipos de incidentes ${ }^{4}$.

Este tipo de trauma tem um agravante no prognóstico das vítimas, já que o Sistema Nervoso Central (SNC) possui uma ampla vulnerabilidade à agressão e sua capacidade de recuperação é limitada ${ }^{5}$. Sendo assim, as vítimas que resistem ao TCE podem apresentar deficiências e incapacidades temporárias ou permanentes, que podem interferir na habilidade do indivíduo em exercer suas funçóes ${ }^{6}$.

A evolução do usuário dos serviços de saúde com TCE necessita ser acompanhada e registrada de maneira sistemática e objetiva. Assim, a utilização da avaliação neurológica é fundamental, pois permite explorar e perceber mudanças do estado clínico. Essa avaliação deve ser realizada periodicamente, especialmente na fase aguda, para a detecção precoce das alteraçóes neurológicas, prevenindo assim um mau prognóstico para o paciente ${ }^{7}$.

O enfermeiro de UTI, enquanto integrante da equipe multiprofissional em saúde, possui papel fundamental na atenção aos usuários vítimas de TCE. Além de todas as práticas inerentes ao profissional inserido neste setor hospitalar, o cuidado da vítima de TCE requer atenção específica à manutenção de parâmetros neurológicos (consciência, sensibilidade e motricidade) e fisiológicos diversos (pressão sanguínea, saturação de oxigênio, pressão de perfusão cerebral), na perspectiva de prevenção ou tratamento precoce de lesóes encefálicas secundárias ao traumatismo em ato. Com isso, a avaliação neurológica desses sujeitos torna-se um elemento imprescindível no cotidiano das práticas de enfermagem em $\mathrm{UTI}^{8}$.
Neste sentido, o presente trabalho teve como objetivo analisar a compreensão dos enfermeiros que atuam em UTI acerca da avaliação neurológica em vítimas de TCE, bem como suas estratégias e dificuldades na execução da mesma. Deste modo, é imprescindível que o enfermeiro possua conhecimentos relacionados à neurologia com o intuito de promover cuidados qualificados de enfermagem.

\section{MÉTODO}

Trata-se de uma pesquisa descritiva, com abordagem qualitativa, que foi desenvolvida no Hospital Regional Tarcísio Maia (HRTM) em Mossoró-RN/Brasil. O estudo foi construído a partir do discurso de sete enfermeiros que atuam na UTI da referida instituição. Os critérios de inclusão no grupo estudado restringiram-se a fazer parte do quadro de enfermeiros efetivos UTI e, acima de tudo, possuir experiência profissional igual ou superior a cinco anos. Como critério de exclusão, tinha-se estar afastado por férias ou algum tipo de licença, assim como possuir experiência profissional inferior ao período estipulado.

As informaçôes foram coletadas a partir de entrevistas semiestruturadas, compostas por perguntas abertas que tratavam do cotidiano das práticas de enfermagem voltadas para avaliação neurológica de vítimas com TCE. Além disso, buscava levantar os critérios que os próprios enfermeiros julgavam indispensáveis no que se refere à avaliação neurológica, assim como buscava desvendar possíveis dificuldades intrínsecas a este processo. Os dados foram coletados entre os meses de Janeiro e Março de 2011 e nos resultados do artigo são destacadas as perguntas abertas que foram realizadas.

Depois de coletados, os dados foram transcritos e discutidos com base na técnica de análise de conteúdo. Tal recurso pode ser definido como um conjunto de técnicas que busca obter, por intermédio de estratégias objetivas e sistemáticas, a descrição dos conteúdos inerentes às mensagens que permitam a inferência de conhecimentos relativos às condiçóes de produção/recepção destas mensagens ${ }^{9}$.

A pesquisa foi desenvolvida respeitando os princípios Éticos preconizados pela Resolução 196/96 do Conselho Nacional de Saúde - CNS e os dados somente 
foram coletados após aprovação no Comitê de Ética da Faculdade de Enfermagem Nova Esperança - FACENE (Protocolo no 107/2010 e CAAE n 2973.0.000.351-10).

\section{RESULTADOS}

Os resultados mostraram que dos sete enfermeiros entrevistados, seis afirmaram que realizavam avaliação neurológica nos usuários com TCE e apenas um enfermeiro negou que essa prática fosse realizada pela Enfermagem, ressaltando que apenas o profissional médico era o responsável por esse exame e que, em algumas situações, preferia que outros profissionais não se envolvessem no caso. Além disso, foi possível identificar nos discursos que a maioria dos enfermeiros entrevistados restringia a avaliação neurológica ao uso da Escala de Coma de Glasgow (ECGl), assim como reconheciam a existência de dificuldades no processo de avaliação neurológica (Tabela 1).

\section{DISCUSSÃO}

A avaliação neurológica realizada pelo enfermeiro no âmbito da UTI tema a finalidade de identificar os sinais e sintomas relacionados ao desequilíbrio de funçóes neurológicas e quais serão as suas implicações sobre as necessidades básicas do sujeito. Esse planejamento das açóes de enfermagem contribui para uma melhoria da qualidade da assistência às vítimas com TCE, bem como

Tabela 1

Apresentação das perguntas abertas realizadas no ato da entrevista, seguidas dos principais discursos obtidos

\begin{tabular}{|c|c|}
\hline Perguntas & Principais respostas \\
\hline $\begin{array}{l}\text { Com relaçáo à avaliaçáo } \\
\text { neurológica aplicada às vítimas } \\
\text { de TCE, você a desempenha } \\
\text { nesses casos? }\end{array}$ & $\begin{array}{l}\text { O neurológico a gente nunca pode deixar de lado nem em pacientes de outras patologias. } \\
\text { (...) Mas quando eu já sei que é uma vítima de TCE, já chega com diagnóstico fechado, } \\
\text { a gente dá uma avaliação mais completa. Mais especial, já que é um paciente neurológico } \\
\text { (...). E1 }\end{array}$ \\
\hline & $\begin{array}{l}\text { A avaliação neurológica é mais feita pelo médico, que ele vem todo dia. (...) porque ele } \\
\text { nem quer que a gente se envolva muito com os pacientes deles (...). Essa avaliaçâao náo é } \\
\text { feita por nós, é por ele. E5 }\end{array}$ \\
\hline
\end{tabular}

Com relaçáo à avaliaçáo neurológica desempenhada por você, quais as suas etapas? Como você a organiza?
Utilizamos Escala de Glasgow. Então é muito importante fazer no tratamento do paciente essa escala de Glasgow, porque se o paciente estiver com escore menor ou igual a oito já é indicação de intubação, se ele não estiver intubado, você tem que preservar essa via aérea, porque se ele não tiver esses cuidados prioritários ele pode broncoaspirar, e pode evoluir para uma pneumonia por broncoaspiração. E1

Enfatizamos a Escala de Coma de Glasgow, pois dependendo da graduação a gente vai tomar o direcionamento da assistência de Enfermagem. Se o Glasgow tiver igual ou inferior a 8 a gente vai ter um trabalho em equipe, comunicar ao médico, para ver se é necessário uma intubação, suporte ventilatório, e assim sucessivamente (...). E2

Você tem que avaliar o nivel de consciência do paciente, você tem que avaliar os reflexos, você não pode isolar essas etapas. Para o caso desse paciente, naquele momento, todos são importantes. E6

Avalio o nivel de consciência, se ele vai apresentar alguma alteração, como, por exemplo, algum sinal, uma otorragia (...), as pupilas a gente tem que ter cuidado com elas. Se estão anisocóricas, se estão em midríase (...). E7

\footnotetext{
No que se refere à avaliçáo neurológica, quais as dificuldades que você encontra para a sua execuçáo?
}

Na efetivação da avaliação, não encontro dificuldades. Encontro mais dificuldades na efetivação dos cuidados que a gente orienta para que sejam prestados... Muitas vezes, nós colocamos por escrito e a equipe náo os efetivam (...). E2

Sim, tenho muitas dificuldades, porque na prática priorizamos outros sistemas, como o cardiopulmonar. Por mais que tenhamos muitos pacientes neurológicos, sinto que falta conhecimento para prestar assistência adequada (...). E4 
possibilita um cuidado mais individualizado ${ }^{10,11}$.

Porém, a cultura hegemônica do modelo biomédico avigora as especialidades e a centralidade na prática médica (diagnóstico e prescrição terapêutica médica) como sendo o padrão de atendimento proporcionado aos usuários. A consequência desse ato implica na precária valorização e integração de outros profissionais ao cuidado prestado, sempre marginais ou secundários às ações médicas ${ }^{12}$.

A Enfermagem precisa romper com essa lógica e fazer valer seus processos de trabalho aplicados ao trabalho coletivo em saúde, pois a profissão detém vários instrumentos para aplicar em seu exercício profissional, como, por exemplo, o Processo de Enfermagem que é o método recomendado para prática da sua assistência e proporciona autonomia do Enfermeiro, possibilitando-o a intervir nos cuidados prestados aos usuários, reconhecendo-se enquanto coparticipe e corresponsável na elaboração de uma estratégia terapêutica coletiva ${ }^{13}$.

Convém ressaltar que, na assistência em equipe, o cuidado deve ser centrado no usuário dos serviços de saúde e em suas necessidades e não nas categorias profissionais, pois a heterogeneidade de circunstâncias demanda respostas imediatas, sob pena de expor a vida do paciente a riscos. Portanto, a integração dos profissionais na busca de um objetivo comum torna-se essencial para que a assistência ao paciente seja adequada e livre de riscos ${ }^{12}$. Experiências outras têm mostrado que um trabalho transdisciplinar colabora sobremaneira para a recuperação dos sujeitos, uma vez que a unificação dos objetivos favorecem à potencialização das estratégias de cuidado ${ }^{14,15}$.

Dentre aqueles enfermeiros que desempenhavam a avaliação neurológica, foi perceptível que o parâmetro privilegiado pelos profissionais era a ECGl, que se trata de item crucial na avaliação neurológica, pois determina a profundidade e duração da diminuição da consciência em sujeitos que, por exemplo sofreram TCE, utilizando critérios com base fisiológica (abertura ocular, melhor resposta verbal e melhor resposta motora) em que os escores podem variar entre uma faixa de $3-15^{16}$. Ressalta-se que os valores mais altos refletem um quadro menos grave e a medida que esse escore cai, o quadro neurológico do usuário também se agrava ${ }^{17}$.

De fato, a ECGl é uma ferramenta utilizada para realizar a avaliação do nível de consciência, entretanto, não deve ser considerado um exame neurológico completo. Além dela, também se deve contemplar a avaliação das funçôes pupilar, motora, sensitiva, cerebelar e dos nervos cranianos, constituindo, assim, uma avaliação completa da função neurológica ${ }^{18}$.

Levando-se em conta a gama de aspectos que necessitam ser avaliados, observa-se que uma avaliação com fundamentos apenas na ECGl torna-se superficial, pois a mesma permite apenas identificar o nível de consciência dos usuários, o que limita a avaliação quando o examinador tem a pretensão de analisar a gravidade da lesão e seus danos a saúde do paciente. Fato este que está presente no lócus estudado.

Todos esses critérios a serem avaliados, em uma vítima de TCE, darão ao profissional as informaçóes sobre que possível área cerebral foi afetada, quais os danos que esse trauma causou ao sujeito e que cuidados oferecer ao mesmo mediante as manifestações clínicas que este apresenta. Por essa razão, torna-se necessário que o enfermeiro trabalhe uma avaliação completa, na busca de obter melhores resultados na sua prática cotidiana ${ }^{5,8}$.

Vale ressaltar que outro aspecto importante é a padronização desse exame, para que todos os enfermeiros sigam um protocolo no momento da avaliação, pois, foi perceptível a falta desta por parte dos Enfermeiros, transformando a assistência em procedimentos tipicamente individuais. Sugere-se que esta falta de padronização seja reflexo, inclusive, da carência de conhecimentos mais aprofundados acerca da avaliação neurológica, como pode ser vislumbrado em um discurso no qual o enfermeiro aponta a rotina do serviço como vetor na priorização de outros sistemas orgânicos, mesmo quando o sujeito possui comprometimentos na função neurológica, o que repercute em práticas tecnicistas, acríticas, centradas exclusivamente na execução de um procedimento.

É necessário, portanto, apostar na planificação de projetos terapêuticos individuais que coloquem o sujeito e suas necessidades enquanto centro das práticas de um cuidado transdisciplinar e não os profissionais de saúde, cada um com o seu lócus de atuação ${ }^{19}$.

Por fim, o cuidado em enfermagem neurológica demanda um amplo conhecimento por parte dos profissionais que prestam à assistência. Neste sentido, o Enfer- 
meiro deve estar capacitado e ser prudente, pois cuidados impróprios induzem a uma piora do quadro geral do usuário ${ }^{8}$. Certamente, o conhecimento materializado na geração de dispositivos que facilitem a assistência consiste na maior ferramenta para que haja a transformação de atitudes e realidades ${ }^{20}$.

\section{CONCLUSÃO}

De fato, os resultados apontados no estudo sugerem a limitação do conhecimento acerca da avaliação neurológica aplicada às vítimas de TCE's, por parte dos enfermeiros do setor estudado. Limitaçóes estas evidenciadas pela restrição da avaliação desempenhada, pela falta de parâmetros avaliativos e pela quase inexistente padronização das condutas.

Considerando que apenas um setor de uma unidade de saúde foi estudado e, sobretudo, o número restrito de participantes, outras pesquisas serão necessárias para que haja a confirmação dos resultados supracitados e, entâo, seja possível planificar mecanismos de intervenção que vislumbrem a transformaçáo da realidade estudada.

\section{REFERÊNCIAS}

1.Tepas JJ, DiScala C, Ramenofsky ML, Barlow B. Mortality and head injury. J Pediatr Surg 1990;25:92-5. http://dx.doi.org/10.1016/S0022-3468(05)80170-8

2.Mackenzie EJ. Epidemiology of injuries: current trends and future challenges. Epidemiol Rev 2000;22:112-9.

http://dx.doi.org/10.1093/oxfordjournals.epirev.a018006

3.Cavalcanti JRLP, Oliveira FCC, Araújo DP, Gúzen FP. Nursing and head injury: literature systematic review study. Rev EnfUFPE on line 2011;5:128-33. http://dx.doi.org/10.5205/reuol.1320-10510-1-LE.0501201117

4.León-Carrión J, dominguez-Morales MR, Martín JMB, Murillo-Cabezas F. Epidemiology of traumatic brain injury and subarachnoid hemorrhage. Pituitary 2005;8:197-202.

http://dx.doi.org/10.1007/s11102-006-6041-5
5.Nitrini R, Bacheschi L. A. A neurologia que todo médico deve saber. 2. ed. São Paulo: Atheneu; 2005.

6.Sousa RM, Regis FC, Koizumi MS. Traumatismo crânio-encefálico: diferenças das vítimas pedestres e ocupantes de veículos a motor. Rev Saúde Pub 1999;33:85-94.

\section{http://dx.doi.org/10.1590/S0034-89101999000100011}

7.Qureshi AA, Mulleady V, Patel A, Porter KM. Are we able to comply with the NICE head injury guidelines? Emerg Med J 2005;22:861-2.

http://dx.doi.org/10.1136/emj.2004.018481

8.McNett M, Doheny M, Sedlak CA, Ludwick R. Judgments of critical care nurses about risk for secondary brain injury. Am J Crit Care 2010;19:250-60. http://dx.doi.org/10.4037/ajcc2009293

9.Bardin L. Análise de Conteúdo. Lisboa: Ediçôes, 1979, 70p.

10.Cintra EA, Nishide VM, Nunes WA. Assistência de Enfermagem ao paciente crítico. São Paulo: Atheneu; 2000, 671p.

11.Feitoza DS, Freitas MC, Silveira RE. Traumatismo cranioencefálico: diagnósticos de Enfermagem a vítimas atendidas em UTI. Rev Eletr Enf 2004;6:223-33.

12.Alves M, Ramos FRS, Penna CMM. O trabalho interdisciplinar: aproximaçôes possíveis na visão de Enfermeiras de uma unidade de emergência. Texto Contexto Enf 2005;14:323-31.

13. Oliveira SCC, Oliveira FCC, Alves TEA, Soares FRR, Cavalcanti JRLP. Nursing assistance to the victims with traumatic brain injury: (re)discussing the emergency practices. Rev enferm UFPE on line 2011;5(10):2415-19.

http://dx.doi.org/10.5205/reuol.2133-15571-1-LE.0510201111

14.Eifert B, Maurer-Karattup P, Schorl M. Integration of intensive care treatment and neurorehabilitation in patients with disorders of consciousness: a program description and case report. Arch Phys Med Rehab 2013;1-10.

15.Hudson CM, Adams D, DeRose A, Harro CC. Taking an interdisciplinary team approach to rehabilitation after TBI. Nursing 2008;38:7-11.

http://dx.doi.org/10.1097/01.NURSE.0000343495.84713.13

16. Teasdale G, Jennett B. Assessment of coma and impaired consciousness. A pratical scale. Lancet 1974;2:81-4.

http://dx.doi.org/10.1016/S0140-6736(74)91639-0

17.Alves D, Koizumi MS. Escala de Coma de Glasgow: tempo de reavaliar seu uso em serviços de emergência. Acta Paul Enf 1999;12:92-100.

18.Barros ALBL (ed.). Anamnese e exame físico: avaliação diagnóstica de Enfermagem no adulto. Porto Alegre: Artmed, 2002, 440p.

19.Merhy EE. Apostando em projetos terapêuticos cuidadores: desafios para a mudança da escola médica ou utilizando-se da produçáo dos projetos terapêuticos em saúde como dispositivo de transformação das práticas de ensino-aprendizagem que definem os perfis profissionais dos médicos (Endereço na Internet). Rio de Janeiro: Universidade Federal Fluminense(atualizado em 2010, acessado em 2010). Disponivel em:: http://www.uff.br/saudecoletiva/ professores/merhy/artigos-07.pdf.

20.Amante LN, Rossetto AP, Schneider DG. Sistematizaçáo da assistência de enfermagem em unidade de terapia intensiva sustetada pela teoria de Wanda Horta. Rev Esc Enf USP 2009; 43:54-64.

http://dx.doi.org/10.1590/S0080-62342009000100007 